ISSN: 2578-3335 (Print) 2578-3343 (Online)

\title{
Multidisciplinary Approach to Diagnosing and Managing a Case of Peritoneal Mesothelioma
}

Jonathan Maw D.O.

Los Angeles County+USC Medical Center, j.maw24@gmail.com

Geoffrey Kelly M.D. Candidate

Cooper Medical School of Rowan University, kellyg61@rowan.edu

Miriam Enriquez M.D.

Cooper University Hospital, eniriquez-miriam@cooperhealth.edu

Ronak Gor D.O.

Cooper University Hospital, gor-ronak@cooperhealth.edu

Pauline Germaine D.O.

Cooper University Hospital, germaine-pauline@CooperHealth.edu

Cooper Rowan Medical Journal: https://rdw.rowan.edu/crjcsm

Would you like to be a reviewer? Please fill in this short form to express your interest.

\section{Recommended Citation}

Maw, Jonathan D.O.; Kelly, Geoffrey M.D. Candidate; Enriquez, Miriam M.D.; Gor, Ronak D.O.; and Germaine, Pauline D.O. (2020) "Multidisciplinary Approach to Diagnosing and Managing a Case of Peritoneal Mesothelioma," Cooper Rowan Medical Journal: Vol. 2 : Iss. 1 , Article 6.

DOI: 10.31986/issn.2578-3343_vol2iss1.6

Available at: https://rdw.rowan.edu/crjcsm/vol2/iss1/6

\section{(c) (i)}

This work is licensed under a Creative Commons Attribution 4.0 License.

This Case Reports and Case Series is brought to you for free and open access by the Rowan University Journals at Rowan Digital Works. It has been accepted for inclusion in Cooper Rowan Medical Journal by an authorized editor of Rowan Digital Works. For more information, please contact brush@rowan.edu. 


\section{Multidisciplinary Approach to Diagnosing and Managing a Case of Peritoneal}

Mesothelioma 


\title{
Multidisciplinary Approach to Diagnosing and Managing a Case of Peritoneal Mesothelioma
}

\author{
Jonathan Maw, D.O. ${ }^{1 *}$, Geoffrey Kelly, M.D. Candidate ${ }^{2}$, Miriam Enriquez, M.D. ${ }^{3}$, \\ Ronak Gor, D.O. ${ }^{4} \&$ Pauline Germaine, D.O. ${ }^{5}$
}

${ }^{1}$ Radiology Residency, Los Angeles County+USC Medical Center, Los Angeles, California, United States ${ }^{2}$ Cooper Medical of Rowan University, Camden, NJ, United States

${ }^{3}$ Pathology, Cooper University Hospital, Camden, NJ, United States

${ }^{4}$ Surgery and Urology, Cooper University Hospital, Camden, NJ, United States

${ }^{5}$ Radiology, Cooper University Hospital, Camden, NJ, United States

*Corresponding author: j.maw24@gmail.com (Jonathan Maw, D.O.)

\begin{abstract}
Peritoneal mesothelioma is a rare cause of a peritoneal mass in adults and can occur in malignant or benign forms. Compared to the pleural variant of mesothelioma, the peritoneal form is understudied due to the small number of reported cases. We present a case of an 84-year-old male with a history of asbestos exposure who initially presented for an aggravated hernia, was found to have an incidental mass on imaging, and ultimately was diagnosed with malignant peritoneal mesothelioma (MPM) ${ }^{1}$ likely related to prior asbestos exposure. This case study will provide a review of literature and discuss the role of imaging for MPM.
\end{abstract}

Keywords: malignant peritoneal mesothelioma, asbestosis, pleural mesothelioma, imaging in peritoneal mesothelioma, MPMCT index, cytoreductive surgery, hyperthermic intraperitoneal chemotherapy

\section{INTRODUCTION}

Malignant peritoneal mesothelioma is an extremely rare form of malignant mesothelioma. Malignant mesotheliomas are neoplasms that arise from mesothelial cells or the serosal mesenchymal cells of the pleura, peritoneum, pericardium, or tunica vaginalis of the testis; the pleural variant is the most common ${ }^{1}$. Out of approximately 3300 cases of mesothelioma diagnosed in the US every year, about 10-15 percent are of peritoneal origin ${ }^{2,3}$. Studies have shown that of the $\sim 3000$ reported diagnosed pleural mesothelioma cases per year in the US from 2003 to 2008, there is a significant predominance of men diagnosed. In 
contrast, of the 300-400 newly diagnosed cases of MPM per year, the prevalence between men and women is approximately equal ${ }^{3}$. With the exception of asbestos exposure as a predominant risk factor, there is unclear evidence whether the molecular pathogenesis of peritoneal carcinoma is similar to that of pleural mesothelioma. Studies showing different gene expression profiles of pleural and malignant mesotheliomas suggest differences ${ }^{4}$. Since it is an extremely rare disease, there is no data available from randomized controlled studies regarding the imaging findings and treatment options for MPM. Thus, there is significant value in studying individual cases involving such a rare disease with limited data available.

\section{Case report}

An 84-year-old male with a medical history of colon cancer, COPD, and a ventral midline hernia initially presented to the emergency department (ED) after he aggravated the hernia by pulling a plant out of his yard. Upon presentation, the patient's symptoms were unimpressive except for pain from the hernia. Physical examination was positive for an approximately $3 \mathrm{~cm}$ midline epigastric hernia with no abdominal distension. Abdomen was only tender to palpation in the area of the hernia. Initial computed tomography (CT) abdomen and pelvis was notable for an incidental $8.2 \times 3.9 \times 3.3 \mathrm{~cm}$ pelvic mass abutting the bladder and sigmoid colon (Figures 1 and 2), mild ascites, a mildly thickened overlying peritoneum, and a small region of nodular peritoneal thickening. Subsequent colonoscopy was done to rule out a gastrointestinal primary as the source of the mass and showed no extrinsic compression/evidence of disease recurrence of colon cancer. Three months following the colonoscopy, MRI pelvis without and with contrast showed further enlargement of the complex, solid and cystic mass in the pelvis displacing nearby structures (Figures 3 and 4). Tissue sampling was performed via ultrasound-guided biopsy utilizing a 20-gauge needle. In addition, $100 \mathrm{cc}$ of gelatinous material was aspirated. Cytology results of the gelatinous material showed clusters of proliferative mesothelial cells. However, the specimen results from core needle biopsy were inconclusive. Subsequently, the patient transferred his care to a tertiary center where review of previously obtained imaging was performed, highlighting presence of calcified and noncalcified pleural plaques at the lung bases. Review of social history during the multidisciplinary tumor board discussion confirmed prior asbestos exposure. This occupational exposure history, along with the colonoscopy showing no evidence of primary colon cancer, raised the concern for malignant mesothelioma as a possible etiology of the pelvic mass. It was decided surgical management was the best approach to obtain definite tissue diagnosis and alleviate regional mass effect. Preoperative CT urogram revealed further enlargement of the pelvic mass, with lateral deviation and decreased caliber of both ureters as well as compression and anterior displacement of the bladder by the mass (Figure 5).

Two weeks after tumor board, the patient was ultimately taken to the operating room and underwent radical en bloc resection of pelvic mass $>10 \mathrm{~cm}$ (Figure 6) with complete colectomy and end-loop 
ileostomy performed by a surgical oncologist as well as ureterolysis by a urologist. Surgical pathology revealed a tumor consisting of infiltrating epithelioid cells, arranged in nests, tubules, and papillary-like structures (Figure 7). Immunohistochemistry revealed positive staining for mesothelial markers including calretinin, cytokeratin 5/6, and D240 (Figure 8); immunostains for adenocarcinoma (BerEP4, Tag72) and specifically, colorectal carcinoma markers (CK20, CDX2, SATB2) were negative. The final diagnosis was epithelioid malignant mesothelioma involving the peritoneum, serosal aspect of the colon, and the small bowel.

Patient was then discharged to a long-term acute care hospital (LTACH) with follow-up scheduled with surgical oncology and urology. However, the patient expired about a month after surgery secondary to cardiac arrest at long term acute care hospital.

\section{DISCUSSION}

Clinical Presentation

MPM presents with vague, nonspecific symptoms and imaging findings. Presentation varies from incidental imaging findings with no clinical symptoms to abdominal pain, distension, early satiety, dysphagia, shortness of breath, and weight loss. Abdominal distension is the most common initial symptom; abdominal pain is the second most common ${ }^{5}$. Our patient's only symptom at presentation was abdominal pain, which was focal and attributed to the hernia.

Risk factors

Several risk factors for the development of MPM have been studied. Similar to other types of mesotheliomas, there is an association between asbestos exposure and development of MPM ${ }^{6}$. However, the link between asbestos exposure is not as strong in the development of MPM as compared to pleural mesothelioma, particularly in women ${ }^{7}$ and requires more cumulative asbestos exposure ${ }^{8}$. Studies have also shown an increased risk of developing MPM in patients who received direct peritoneal radiation ${ }^{9}$. In addition, there are some genetic links to MPM. Pleural malignant mesothelioma has been linked to the loss of BAP1, NF2, and CDKN2A tumor suppressor genes. A study showed that loss of BAP1 alone without the loss of NF2 and CDKN2A was linked to MPM, suggesting that pleural and peritoneal mesotheliomas have distinct genomic features ${ }^{10}$.

Pathological Features

MPM can be classified on a macroscopic level as either diffuse or focal. Gross pathology of diffuse MPM appears as multiple grey or white nodules scattered around the peritoneum. Advanced diffuse MPM can spread and encase the peritoneal cavity and intraperitoneal organs, causing the small bowel to become fixed, rigid, and immobile. Focal MPM usually forms a solid mass (Figure 6) but can also form masses 
with cystic and mucoid regions. Diffuse MPM may extend into the retroperitoneum, abdominal wall, or diaphragm and into the pleural cavity ${ }^{1}$. Out of all malignant mesotheliomas, approximately $85 \%$ are diffuse. Resectable, localized cases are rare ${ }^{11}$.

Diagnosis of MPM is made by biopsy. Cytologic analysis of ascitic fluid can be used for diagnosis, but is often inconclusive and has a low yield due to the low number of malignant cells in ascites and the significant cytologic diversity of tumor cells ${ }^{12,13}$. It also does not allow for assessment of true stromal invasion into the peritoneum or viscera, limiting assessment of true malignant extent with invasion. Biopsy of solid tumor in comparison, allows the ability to assess stromal invasion ${ }^{12}$.

Histologically MPM can be divided into three subtypes. Epithelioid subtype carries the best prognosis, while the sarcomatous and mixed subtypes carry a poorer prognosis. The epithelioid subtypes appear most like normal mesothelial cells in a tubulopapillary or trabecular pattern. The sarcomatoid subtype consists of tightly packed spindle cells with occasional presence of malignant osteoid, chondroid, or muscular elements. The biphasic subtype consists of both components with each contributing at least $10 \%$ of the overall histology ${ }^{1}$. Approximately $75 \%$ of MPM are of the epithelioid subtype, while the biphasic subtype is $\sim 25 \%$. The sarcomatoid subtype is extremely rare and is almost $0 \%$ of MPM $^{13}$. Our patient's core-needle biopsy was inconclusive; fluid cytology suggested the diagnosis and the surgical resection ultimately resulted in histologic sections most consistent with the epithelioid subtype (Figure 7). There are no markers that are specific for malignant mesothelioma in immunohistological analysis. However, markers such as calretinin, D240, WT1, and cytokeratin 5/6 are used to help differentiate malignant mesotheliomas from other carcinomas with similar histologic appearance ${ }^{1}$. Figure 8 shows tumor cells with reactivity with calretinin, supporting mesothelial differentiation of the mass in our case. Imaging Features

CT scan is the most common initial imaging modality used in diagnosing MPM as it is usually the initial test ordered in a patient presenting with abdominal pain or increased abdominal girth. There is a wide spectrum of imaging findings in MPM, but results most commonly include a thickening of the mesentery and peritoneum ${ }^{14}$. It can be classified by distinct patterns on cross-sectional imaging that reflect its gross pathologic appearance: diffuse peritoneal cavity involvement or focal intraperitoneal mass. While imaging findings for MPM are variable, diffuse MPM is most commonly characterized as widespread diffuse involvement of the peritoneum with tumor infiltrating and thickening the peritoneum, in a sheet-like fashion, leading to an irregular and nodular pattern. Focal MPM can present as dominant intraperitoneal mass associated with peritoneal studding, sometimes with omental caking. Omental caking can appear as fine, nodular, soft-tissue studding or coalescent, mass-like soft tissue in the omentum. In addition, sometimes calcifications related to the malignant osteoid, chondroid, or muscular elements of the 
sarcomatoid subtype can be seen on CT $\operatorname{scan}^{1}$.

Since CT findings for MPM are highly variable, differentiating MPM from other peritoneal masses such as carcinomatosis, gastrointestinal malignancies, ovarian carcinomas, and lymphomas is difficult. A study that compared the CT findings of MPM with non-MPM peritoneal masses showed three statistically significant characteristics for differentiating MPM from non-MPM masses: multiple nodular lesions, mild to massive thickening of the mesentery, and presence of pleural plaques. While not statistically significant, it also showed the MPM group had a higher proportion of cases with moderate to massive accumulation of ascites, nodular lesions with a maximum dimension of $<1 \mathrm{~cm}$, and irregular or massive thickening of the peritoneum ${ }^{14}$. However, since there is no single imaging finding that is specific to MPM ${ }^{13}$, suspicion of MPM is made from imaging findings in addition to clinical context; final diagnosis is made from biopsy. Studies demonstrate that fat-suppressed and delayed gadolinium-enhanced magnetic resonance imaging (MRI) can be of use to accurately estimate the burden of peritoneal disease because the contrast enhancement allows depiction of sub-centimeter deposits and deposits located in anatomically difficult sites to see on CT, such as the subphrenic, mesenteric areas, and along the bowel serosal surfaces ${ }^{15}$. However, this is not well defined for MPM ${ }^{13}$. MRI is used less commonly than CT for MPM and shows a mass with low-intermediate signal on T1 weighted images, and high signal on T2 weighted images Figure 4 due to internal necrosis ${ }^{16}$. It is most commonly used as a primary imaging modality for MPM for patients who cannot tolerate IV contrast. Similarly, while positive emission tomography (PET) and PET/CT scans are often used to stage cancers, it is unclear if they have any value in staging MPM ${ }^{13}$. Due to small size of peritoneal deposits, they may be associated with low level activity on PET and PET/CT, making assessment of true extent of disease involvement difficult. Localized mass is frequently associated with significant degree of necrosis, further limiting the applicability of PET.

CT images of our patient had all three of the distinguishing findings of MPM including nodular lesions, thickening of the peritoneum, and presence of pleural plaques. MRI confirmed the progressive enlargement of the mass over time; no additional information was revealed by MRI in comparison to the initial CT. Careful review of imaging studies raised the possibility of MPM and guided surgical planning. Treatment

Most research on MPM treatment has been in the form of retrospective single-center series, which is prone to inherent selection biases. Data on systemic chemotherapy is mainly available through Expanded Access Programs (outside of clinical trials). Historically MPM was treated with systemic chemotherapy but with modest survival rates with a median survival rate between 10-26.8 months ${ }^{17}$. Recent advancements have led to treatment with a combination of cytoreductive surgery (CRS) and hyperthermic intraperitoneal chemotherapy (HIPEC), which has shown improved outcomes over surgery or chemotherapy alone ${ }^{17}$. 
Imaging plays a role in management of MPM and can guide surgical planning. Localized MPM has a better prognosis and responds better to surgical resection ${ }^{14}$. The goal of cytoreductive surgery is to remove as much tumor as possible. Chemotherapy can be done systemically or directly into the abdomen (intraperitoneal chemotherapy) after surgery or through an abdominal catheter. Intraperitoneal therapy is considered more effective over systemic therapy because of enhanced concentrations in the area of tumor and decreased systemic toxicity. Studies show the overall response rate with a single agent chemotherapy, combined chemotherapy, intraperitoneal chemotherapy, and continuous hyperthermic peritoneal perfusion are $13.1 \%, 20.5 \%, 47.4 \%$, and $84.6 \%$ respectively ${ }^{18}$. Cisplatin is the most studied chemotherapy agent, and is often used with mitomycin $\mathrm{C}$, fluorouracil, doxorubicin, and/or paclitaxel for intraperitoneal chemotherapy. Cisplatin and pemetrexed are often used in combination for systemic chemotherapy in both pleural and peritoneal mesothelioma. Systemic chemotherapy is more often used in cases where the tumor is unresectable ${ }^{3}$. For our patient, since the final diagnosis was not available and the patient was experiencing lower urinary symptoms, debulking surgery with biopsy was done to provide a diagnosis and alleviate his symptoms. Hence, HIPEC or chemotherapy was not originally considered. Unfortunately, the patient passed away before further treatment could be offered.

\section{CONCLUSION}

In conclusion, MPM is a rare disease that is difficult to diagnose based on its vague symptoms and nonspecific imaging findings. A history of asbestos exposure or pleural plaques should raise the index of suspicion for MPM in evaluation of an abdominal or pelvic mass because early diagnosis and treatment can lead to better outcomes. A multimodality approach is appropriate in the diagnosis and management of MPM. Imaging can lead to suspicion of MPM, show progression of disease, and help guide surgical planning for debulking. Final diagnosis is made by biopsy. The most effective treatment outcomes have resulted from a combination of surgical resection and chemotherapy. 


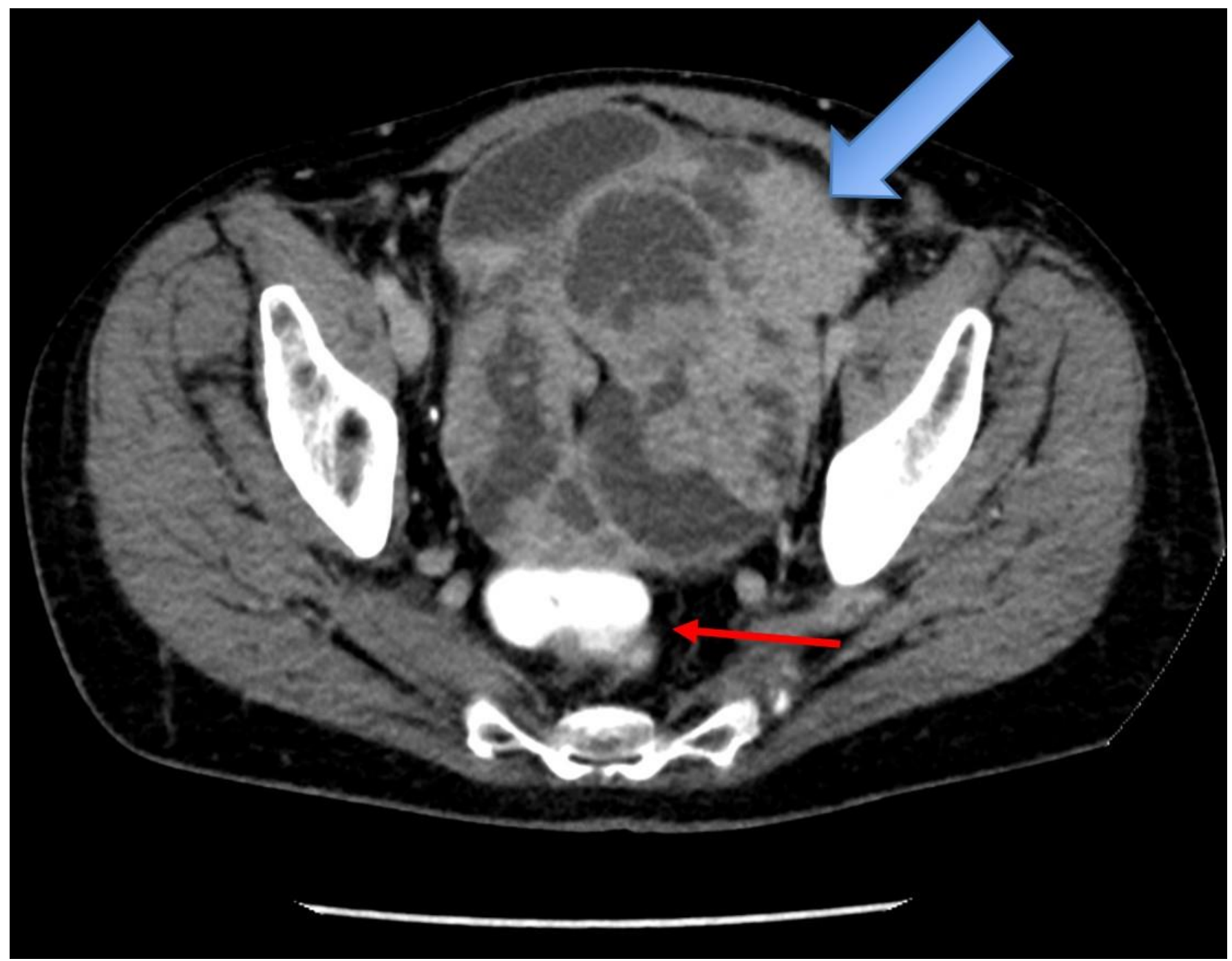

Figure 1 Figures one and two description: Axial and coronal images from the initial CT of the abdomen and pelvis show an incidental heterogeneously enhancing solid and cystic pelvic mass (thick blue arrows)abutting the bladder (yellow triangle) and the rectum (thin red arrow). 


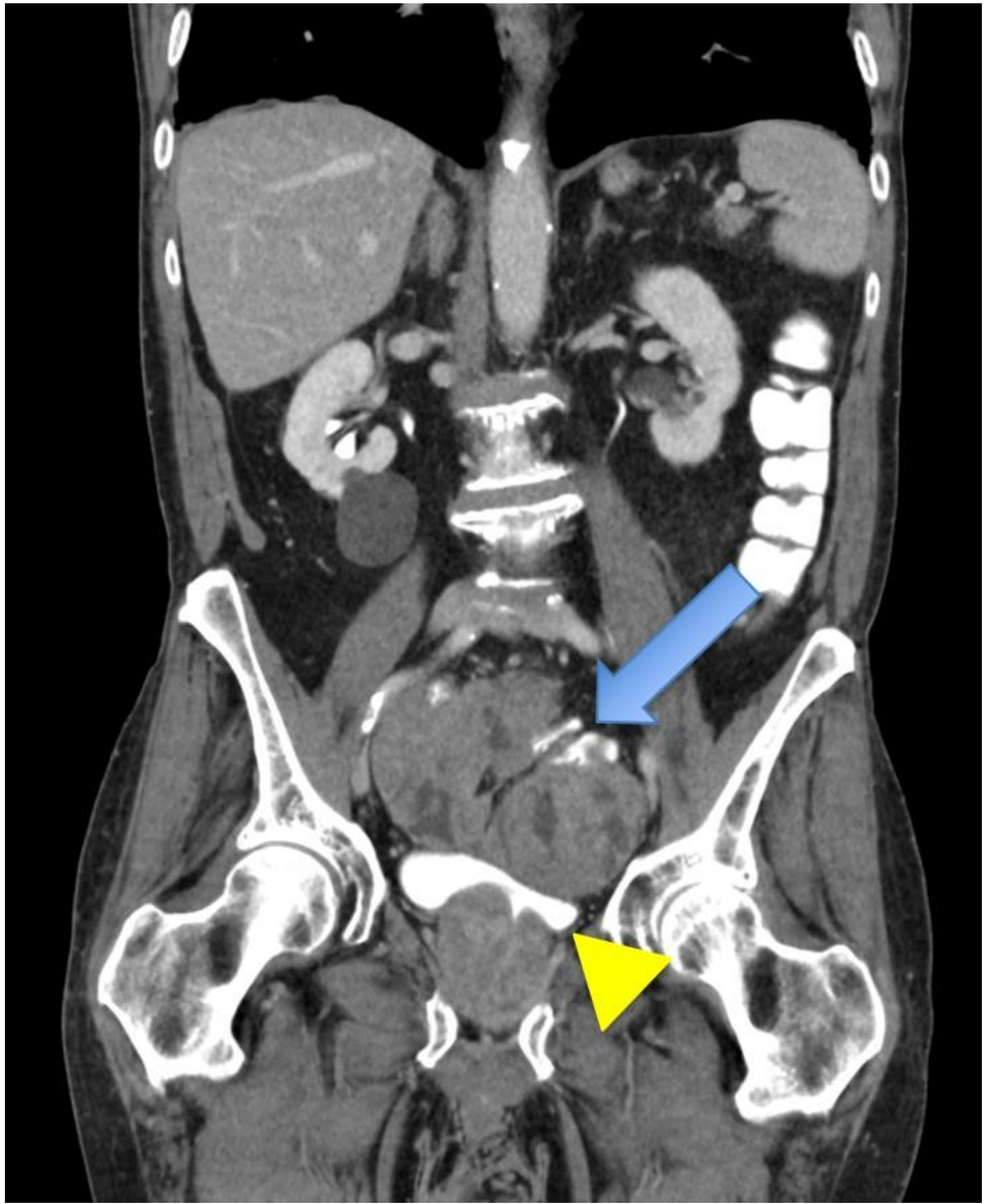

Figure 2 Figures one and two description: Axial and coronal images from the initial CT of the abdomen and pelvis show an incidental heterogeneously enhancing solid and cystic pelvic mass (thick blue arrows)abutting the bladder (yellow triangle) and the rectum (thin red arrow). 


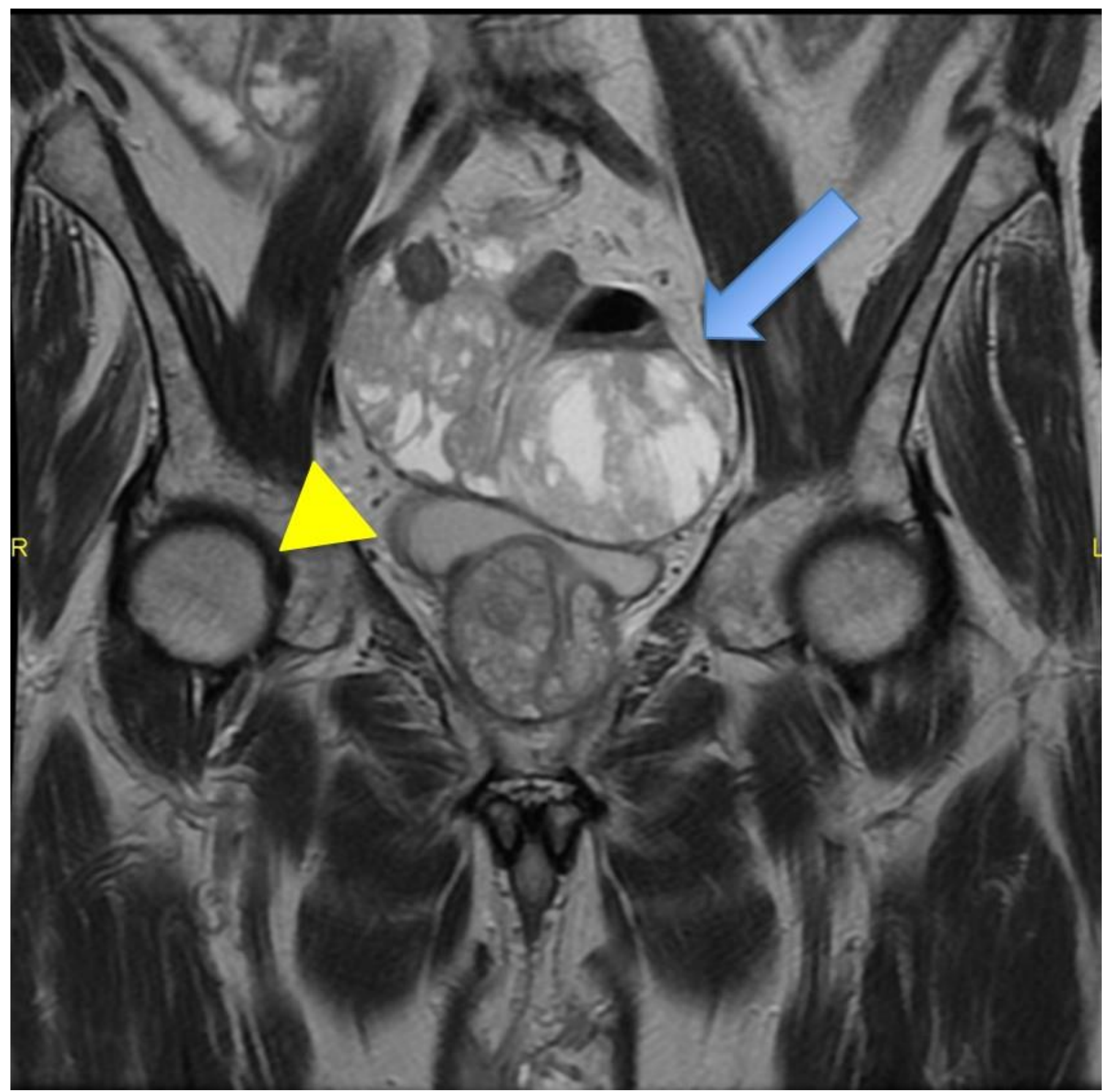

Figure 3 Figures three and four description: Coronal and axial images obtained with echo-planar fast spin echo sequences show further enlargement of complex, solid and cystic mass in the pelvis (blue arrows) displacing nearby structures, including the bladder(yellow triangles). 


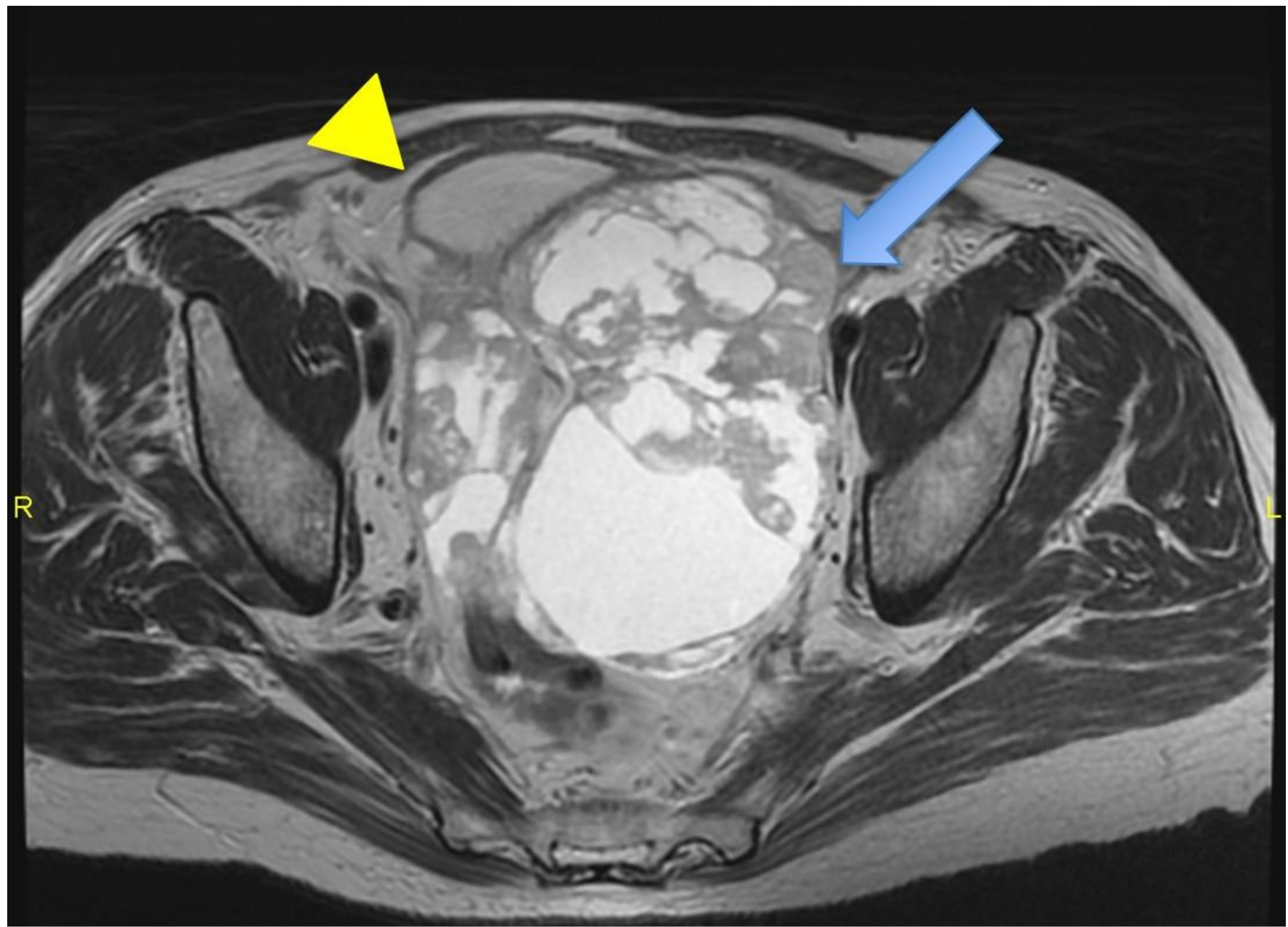

Figure 4 Figures three and four description: Coronal and axial images obtained with echo-planar fast spin echo sequences show further enlargement of complex, solid and cystic mass in the pelvis (blue arrows) displacing nearby structures, including the bladder(yellow triangles). 


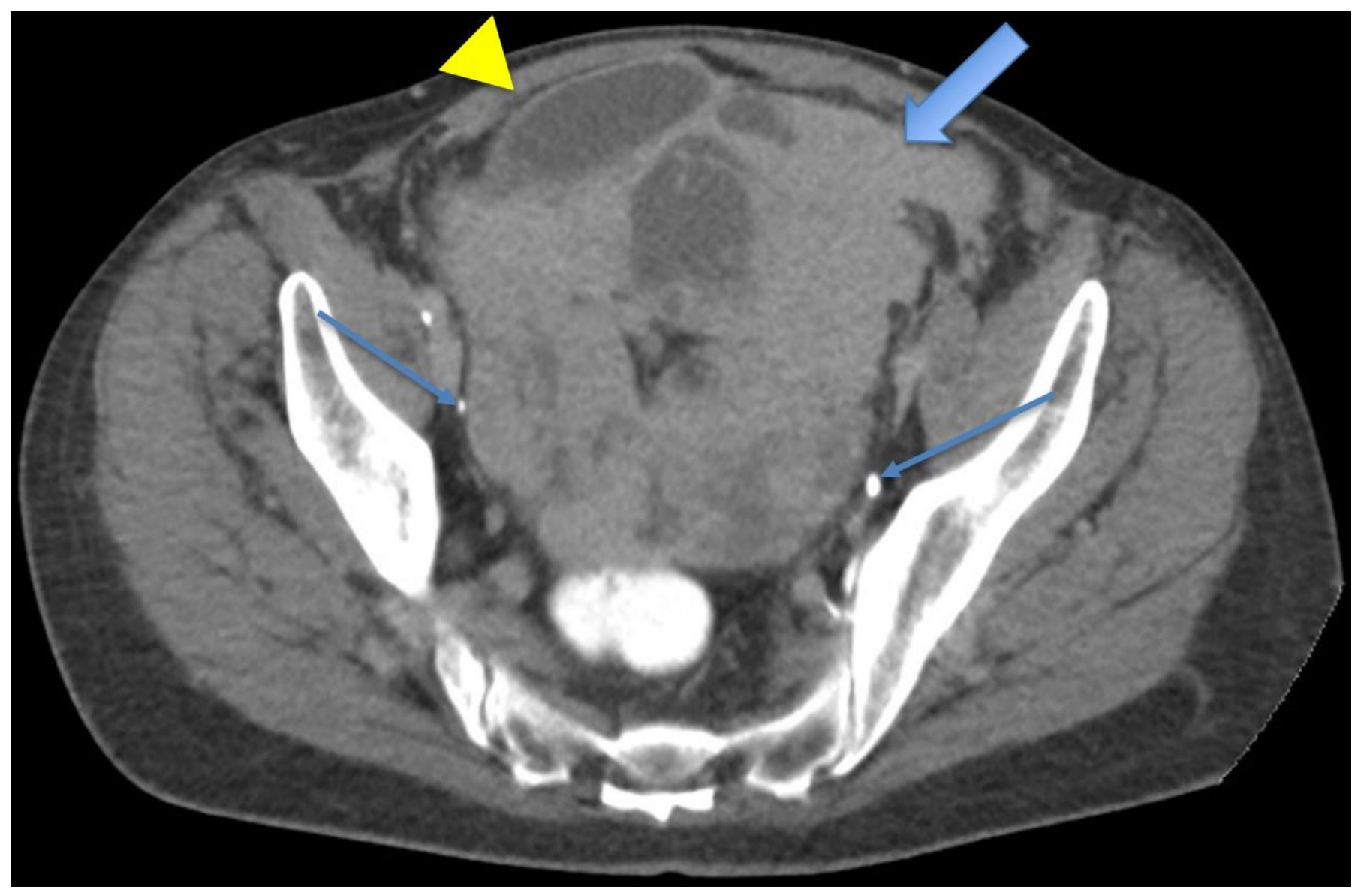

Figure 5 Axial image from preoperative CT urogramshows further enlargement of the pelvic mass (thick blue arrow), with lateral deviation and decreased caliber of both ureters (thin blue arrows) as well as compression and anterior displacement of the bladder by the mass (yellow triangle). 


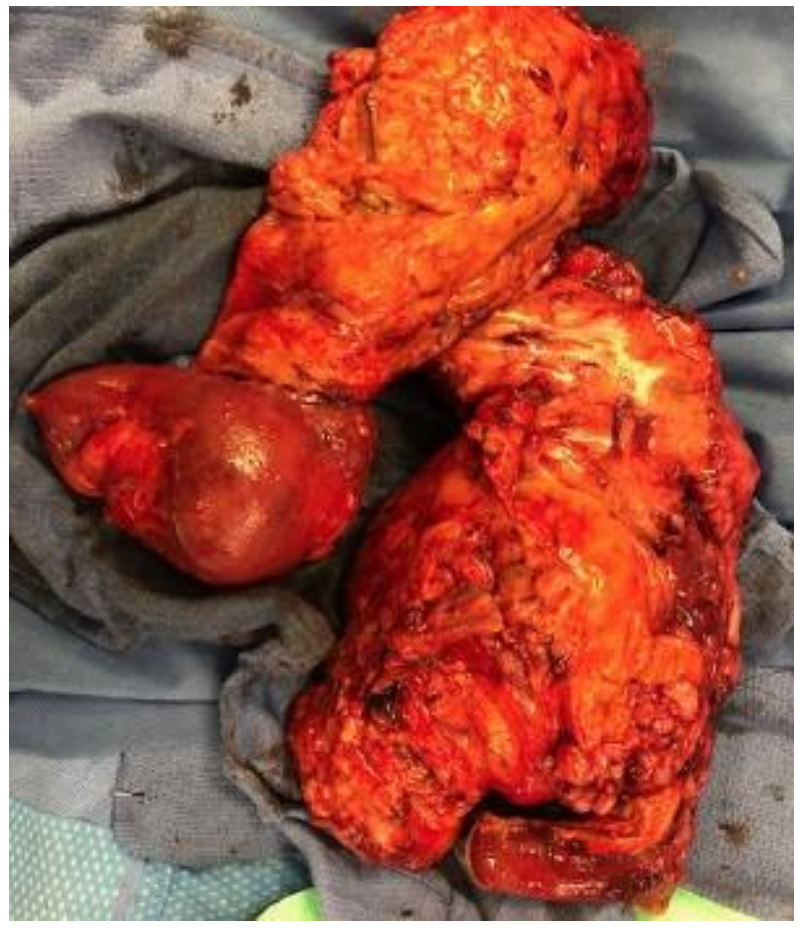

Figure 6 Gross specimen of the pelvic mass from radical en block resection, showing a dominant $22 \mathrm{~cm}$ mass involving the colonic wall, with multiple tumorimplants along the serosal surface and in the mesenteric fat. 


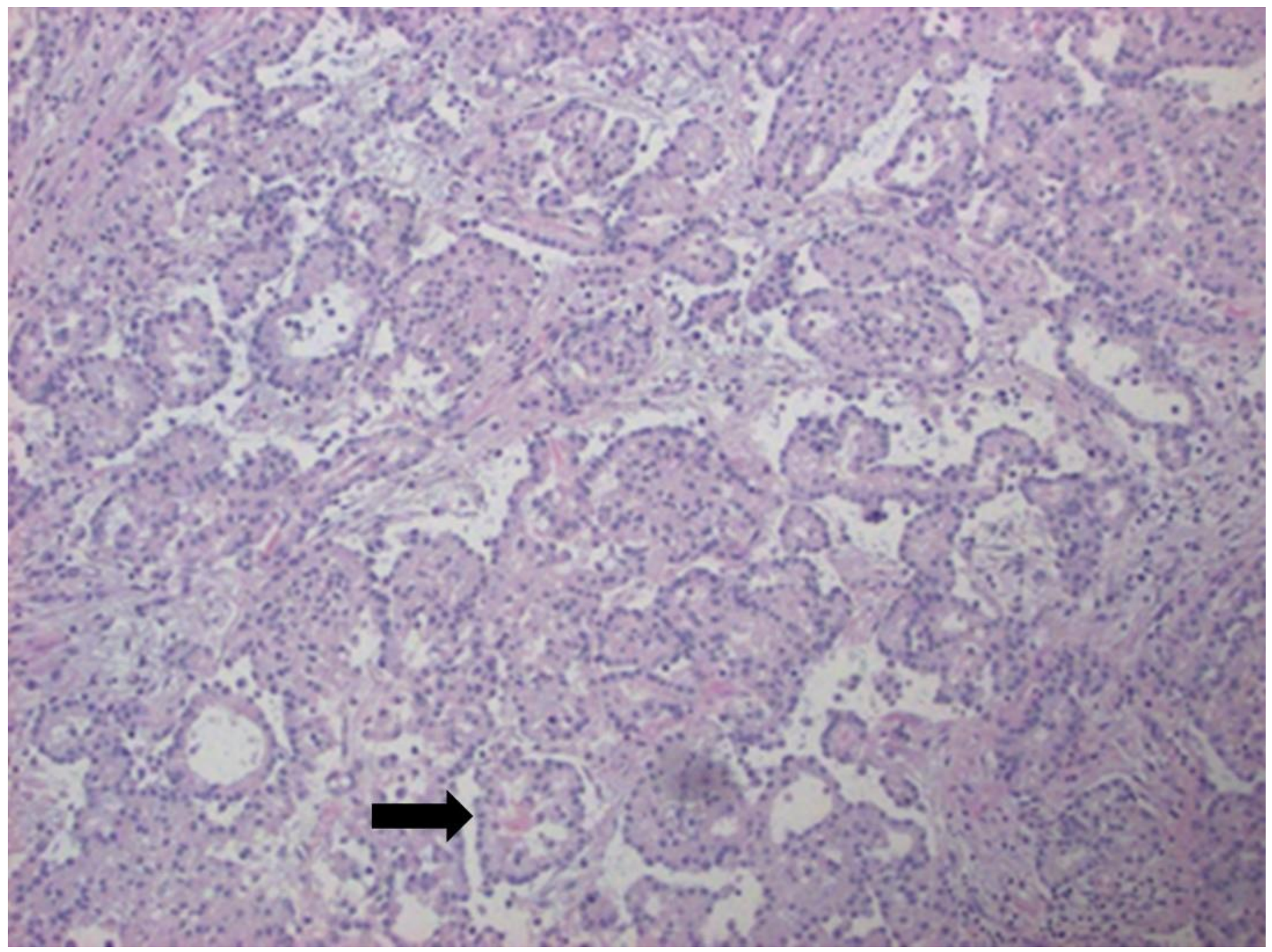

Figure 7 The tumor consists of epithelioid cells arranged variably in nests, tubules, and many papillary-like structures, which are depicted here (black arrow). (H\&E 100x). 
Cooper Rowan M edical Journal, Vol. 2, Iss. 1 [2020], Art. 6

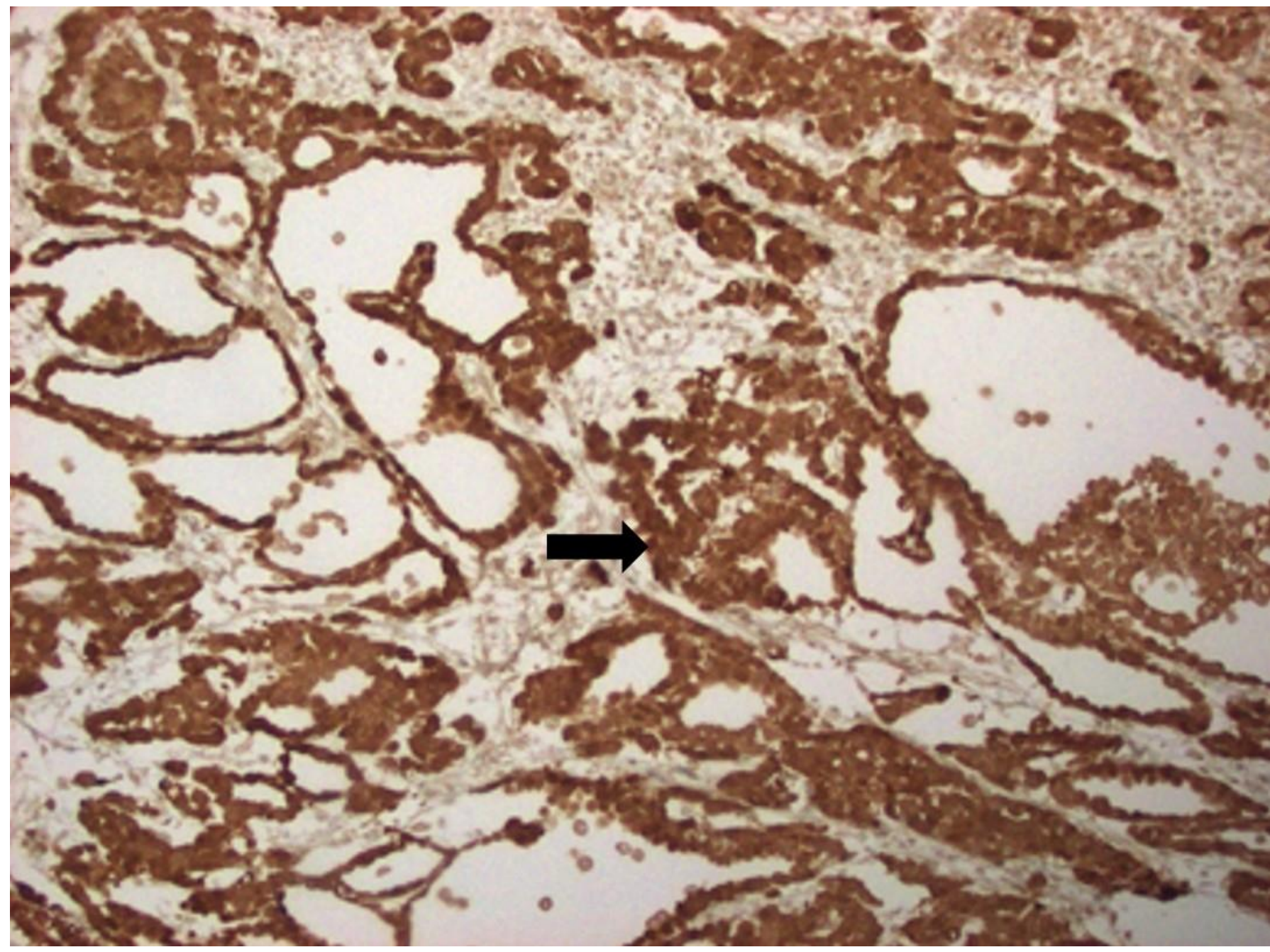

Figure 8 The tumor cells showedstrong immunoreactivity with calretinin (black arrow), supporting mesothelial differentiation (immunostain 100x). 


\section{REFERENCES}

1. Levy A D, Arnáiz J, Shaw J C, Sobin L H. From the archives of the AFIP: primary peritoneal tumors: imaging features with pathologic correlation. Radiographics. 2008;28(2):583-622.

2. Teta Mary Jane, Mink Pamela J., Lau Edmund, Sceurman Bonnielin K., Foster Edward D.. US mesothelioma patterns 1973-2002: indicators of change and insights into background rates. European Journal of Cancer Prevention. 2008;17(6):525-534.

3. Kim Joseph, Bhagwandin Shanel, Labow Daniel M.. Malignant peritoneal mesothelioma: a review. Annals of Translational Medicine. 2017;5(11):236-236.

4. López-Ríos F, Chuai S, Flores R. Global gene expression profiling of pleural mesotheliomas: overexpression of aurora kinases and $\mathrm{P} 16 / \mathrm{CDKN} 2 \mathrm{~A}$ deletion as prognostic factors and critical evaluation of microarray-based prognostic prediction. Cancer Res. 2006;66(6):2970-2979.

5. Acherman Yair IZ, Welch Laura S, Bromley Christina M, Sugarbaker Paul H. Clinical Presentation of Peritoneal Mesothelioma. Tumori Journal. 2003;89(3):269-273.

6. Consonni D, Calvi C, Matteis De, S . Peritoneal mesothelioma and asbestos exposure: a population-based case-control study in Lombardy, Italy. Occup Environ Med. 2019;76(8):545-553.

7. Spirtas R, Heineman E F, Bernstein L, et al. Malignant mesothelioma: attributable risk of asbestos exposure.. Occupational and Environmental Medicine. 1994;51(12):804-811.

8. Hodgson J T, Darnton A. The quantitative risks of mesothelioma and lung cancer in relation to asbestos exposure. Ann Occup Hyg. 2000;44(8):565-601.

9. Farioli Andrea, Ottone Marta, Morganti Alessio G., et al. Radiation-induced mesothelioma among long-term solid cancer survivors: a longitudinal analysis of SEER database. Cancer Medicine. 2016;5(5):950-959.

10. Alakus Hakan, Yost Shawn E, Woo Brian, et al. BAP1 mutation is a frequent somatic event in peritoneal malignant mesothelioma. Journal of Translational Medicine. 2015;13(1):122-122.

11. Allen Timothy Craig, Cagle Philip T, Churg Andrew M, et al. Localized Malignant Mesothelioma. The American Journal of Surgical Pathology. 2005;29(7):866-873.

12. Churg A, Colby T V, Cagle P. The separation of benign and malignant mesothelial proliferations. Am J Surg Pathol. 2000;24(9):1183-1200. 
13. Kim Joseph, Bhagwandin Shanel, Labow Daniel M.. Malignant peritoneal mesothelioma: areview. Annals of Translational Medicine. 2017;5(11):236-236.

14. Kato K, Gemba K, Fujimoto N. Computed Tomographic Features of Malignant Peritoneal Mesothelioma. Anticancer Res. 2016;36(3):1067-1072.

15. Patel Chirag M., Sahdev Anju, Reznek Rodney H.. CT, MRI and PET imaging in peritoneal malignancy. Cancer Imaging. 2011;11(1):123-139.

16. Park J Y, Kim K W, Kwon H J. Peritoneal mesotheliomas: clinicopathologic features, CT findings, and differential diagnosis. AJR Am J Roentgenol. 2008;191(3):814-825.

17. Helm J H, Miura J T, Glenn J A. Cytoreductive surgery and hyperthermic intraperitoneal chemotherapy for malignant peritoneal mesothelioma: a systematic review and meta-analysis.Ann Surg Oncol. 2015;22(5):1686-1693.

18. Hotta T, Taniguchi K, Kobayashi Y. Chemotherapy and serum hyaluronic acid levels in malignant peritoneal mesothelioma. Hepatogastroenterology. 2004;51(58):1073-1083. 